\title{
Optimisation of Hidden Markov Model using Baum-Welch algorithm for prediction of maximum and minimum temperature over Indian Himalaya
}

\author{
J C Joshi ${ }^{1, *}$, Tankeshwar Kumar ${ }^{2}$, Sunita Srivastava ${ }^{2}$ and Divya Sachdeva ${ }^{1}$ \\ ${ }^{1}$ Snow and Avalanche Study Establishment, Sector 37-A, Chandigarh 160 036, India. \\ ${ }^{2}$ Department of Physics, Panjab University, Chandigarh, India. \\ ${ }^{*}$ Corresponding author.e-mail: joshjagdish@gmail.com
}

\begin{abstract}
Maximum and minimum temperatures are used in avalanche forecasting models for snow avalanche hazard mitigation over Himalaya. The present work is a part of development of Hidden Markov Model (HMM) based avalanche forecasting system for Pir-Panjal and Great Himalayan mountain ranges of the Himalaya. In this work, HMMs have been developed for forecasting of maximum and minimum temperatures for Kanzalwan in Pir-Panjal range and Drass in Great Himalayan range with a lead time of two days. The HMMs have been developed using meteorological variables collected from these stations during the past 20 winters from 1992 to 2012. The meteorological variables have been used to define observations and states of the models and to compute model parameters (initial state, state transition and observation probabilities). The model parameters have been used in the Forward and the Viterbi algorithms to generate temperature forecasts. To improve the model forecasts, the model parameters have been optimised using Baum-Welch algorithm. The models have been compared with persistence forecast by root mean square errors (RMSE) analysis using independent data of two winters (2012-13, 2013-14). The HMM for maximum temperature has shown a $4-12 \%$ and $17-19 \%$ improvement in the forecast over persistence forecast, for day-1 and day-2, respectively. For minimum temperature, it has shown $6-38 \%$ and $5-12 \%$ improvement for day-1 and day-2, respectively.
\end{abstract}

\section{Introduction}

The Indian Himalayas has been classified into Lower, Middle and Upper snow climatic zones based on temperature, topography and snowfall during winter. In the Lower Himalayan zone, there are mild temperatures, heavy snowfall and deep snowpack, whereas in Middle and Upper Himalayan zones there are cold temperatures, moderate snowfall and shallow snowpack. Avalanche activities in these regions mainly take place either at the time of snowfall, when temperatures are low or during the spring season, when the temperatures are high, implying that the snow avalanches in these regions are mainly affected by snowfall and temperature. After snowfall, temperature is the most dominant factor contributing towards instability of the snowpack and triggering of avalanches.

Prediction of maximum and minimum temperatures is also important for glaciological and hydrological studies. Snow-melt and glacier-melt are major sources of water, as most of the perennial rivers are fed by snow-melt and glacier-melt in Himalaya (Bolch et al. 2012). The study of snow-melt and glacier-melt for flood forecasting and water resource management relies highly on temperature forecast. Moreover, it is also important

Keywords. Temperature forecast; Hidden Markov Model optimisation; meteorological variables. 
for planning of outdoor activities/operations in snow bound regions.

Variations in maximum and minimum temperatures are caused by various factors including synoptic conditions, seasonal variation and topography of the specific locations. The models forecasting weather variables including temperature can be classified into three groups: Empirical models, statistical models and dynamic models. There is an additional class of models known as stochastic models that include the features of both statistical and dynamical models.

Stochastic and deterministic models have widely been used in the past for forecasting of maximum and minimum temperatures. Woodcock (1984) described some experimental model output statistics (MOS) forecasts of daily maximum and minimum temperatures for seven Australian cities. Raj (1989) evolved a scheme for predicting the minimum temperature at Pune by analogue and regression methods. Mohan et al. (1989) developed a method for forecasting maximum temperature over Ozar, situated in Maharashtra, using the maximum and dew point temperatures of the previous day. Chrantorois and Liakatas (1990) have studied minimum temperature forecasts employing Markov chains. Vashisht and Pareek (1991) have given a partial objective method to forecast minimum temperature at Jaipur and South Rajasthan. Attri et al. (1995) have given a multiple regression method for forecasting the minimum temperature at Gangtok based on knowledge of dew point temperature, cloud amount, maximum and minimum temperature recorded on the previous day. Skill score performances and accuracy of locationspecific temperature forecasts were carried out by Sanjeeva Rao et al. (1996). Kumar and Maini (1996) have carried out statistical interpretation (SI) forecast of GCM to improve location-specific medium range local weather forecast. Mohanty et al. (1997) employed regression techniques for forecasting maximum and minimum temperatures at Delhi during summer and winter seasons, respectively. Raj (1998) employed forecast schemes based upon statistical techniques to forecast daily summer maximum temperature at Chennai. Dimri et al. (2002) developed a statistical model for forecasting maximum and minimum temperatures at Manali using multivariate regression analysis. Maini et al. (2002) have carried out statistical interpretation of T-80 model's output and verified the skill of location-specific forecast for the 1997 monsoon season for a number of stations in India. Dimri et al. (2005) used perfect prognostic method (PPM) for prediction of minimum temperature at Manali, in Pir-Panjal range of Himalayas.

Hidden Markov Models (HMMs) have not only been used in weather prediction, but also used widely in other research fields such as speech pattern recognition (Gales and Young 2007), credit card fraud detection (Bhusari and Patil 2011), face recognition (Bicego et al. 2003), gesture recognition (Tanguay 1995), El Nino studies (Xuan 2004) and prediction of financial time series (Zhang 2004).

Markov models are stochastic models satisfying Markov property. According to this property, the probability of a future state of a process solely depends upon its present state, not on all the previous states. The HMMs are double embedded stochastic processes with two layers - one layer is formed by a state sequence satisfying Markov property and the other by a state-dependent observation sequence. The HMMs are useful to model processes in which there are different states that occur in some order and produce observations.

In the present study, HMMs have been developed for prediction of maximum and minimum temperatures in Lower and Middle snow climatic zones of the Himalaya using surface observations of the past 20 winters (1992-2012) collected from these climatic zones. The models have been developed using nine meteorological variables (maximum temperature, minimum temperature, atmospheric pressure, relative humidity, wind speed, wind direction, cloud amount, cloud type and sunshine hours) selected from the database of large number of variables based on correlation (Joshi and Srivastava 2014) of the variables with their temperature indices. The variables having a good correlation $(>0.25)$ are taken for development of the models.

In Hidden Markov Model, the predicted observation sequence and model parameters are not good many times to explain the observations. So the model parameters are adjusted to maximise the probability of the observation sequence. The HMMs have been optimised using the Baum-Welch algorithm. The root mean square error analysis of the model with and without using optimisation has been carried out using test data of the two winters (2012-14).

\section{Study area and data}

HMM has been developed for forecasting of maximum and minimum temperatures using meteorological data of the past 20 winters (1992-2012). Validation of the models has been done using independent data of two winters (2012-2014). The data recorded at Kanzalwan, a representative observatory in Pir-Panjal range and Drass, a representative observatory in the Great Himalayan range of the Himalaya in J\&K, India, has been taken for model development. The data at these stations is recorded twice daily at 08:30 hours in the morning and 17:30 hours in the evening. The 
long-term mean and standard deviation of maximum and minimum temperature data of Kanzalwan and Drass are shown in table 1.

The meteorological data collected at different observatories of the Snow and Avalanche Study Establishment (SASE) have also been used by other researchers (Mohanty and Dimri ; Dimri et al. 2005; Singh et al. 2005, etc.) for forecasting of temperature and precipitation over Himalaya. The data involved in this study include maximum temperature, minimum temperature, relative humidity, pressure, snowfall amount, snow depth, cloud amount and sunshine hours. Some of the model input data have been derived from the recorded data which includes deviation of minimum temperature from average minimum temperature, deviation of maximum temperature from average maximum temperature, deviation of pressure from the standard atmospheric pressure of the location and deviation of relative humidity from its value at saturation. The rest of the input data such as average wind speed, wind direction, cloud amount, cloud type and sun shine hours have been taken directly from the recorded data. The outliers and missing values have been removed from the data as it affect the model output adversely.

\section{Methodology}

HMMs are based on the sequences of observations and states. There are 10 observations of the models, derived from the weighed sum of temperature index (TI) of all the model input variables, for each station. The variables to be predicted are categorised into 10 categories between their maximum and minimum values to define 10 states of the model. The TI of a category is a ratio of the number of days when temperature was above the mean temperature of the station to the total days in that category. The variables have been correlated with their TI and square of the correlation is used as weight of the variable. The TI is calculated for each of the ranges of all the parameters. Normalisation of the TI of all the parameters has been done between 0 and 1 for making them scale-invariant. Different ranges of observations and state variables of the HMM for maximum and minimum temperatures for both the stations have been summarised in table 2 . The model input variables and their TIs have been plotted in figures 1 and 2. After defining the states and the observations, different probability matrices (initial state probability, state transition probability and probability of observations in different states) have been computed for prediction of maximum and minimum temperatures for both the stations with and without optimisation.

In HMM, there is a state sequence $S_{1}, S_{2}, \ldots, S_{n}$ of ' $n$ ' states and state-dependent observation sequence $O_{1}, O_{2}, \ldots, O_{m}$ of ' $m$ ' observations. The state sequence satisfies the following property of Markov Chain (Rabiner 1989):

$$
\begin{array}{r}
P\left(S_{t} \mid S_{t-1} ; S_{t-2}, S_{t-3}, \ldots\right)=P\left(S_{t} \mid S_{t-1}\right), \\
t=2,3, \ldots
\end{array}
$$

\begin{tabular}{|c|c|c|c|c|}
\hline \multirow[b]{2}{*}{ Stations } & \multicolumn{2}{|c|}{ Maximum temperature $\left({ }^{\circ} \mathrm{C}\right)$} & \multicolumn{2}{|c|}{ Minimum temperature $\left({ }^{\circ} \mathrm{C}\right)$} \\
\hline & Long term mean & Standard deviation & Long term mean & Standard deviation \\
\hline Kanzalwan & 6.3 & 4.0 & -5.8 & 5.2 \\
\hline Drass & 2.3 & 4.2 & -12.6 & 6.2 \\
\hline
\end{tabular}

Table 1. Long term mean and standard deviation of maximum and minimum temperatures.

\begin{tabular}{|c|c|c|c|c|c|}
\hline \multicolumn{3}{|c|}{ Maximum temperature } & \multicolumn{3}{|c|}{ Minimum temperature } \\
\hline \multirow[b]{2}{*}{ Ranges of states } & \multicolumn{2}{|c|}{ Ranges of observations } & \multirow[b]{2}{*}{ Ranges of states } & \multicolumn{2}{|c|}{ Ranges of observations } \\
\hline & Kanzalwan & Drass & & Kanzalwan & Drass \\
\hline $\mathrm{S}_{1}(\leq-6)$ & $\mathrm{O}_{1}(\leq 1.6)$ & $\mathrm{O}_{1}(\leq 1.3)$ & $\mathrm{S}_{1}(\leq-16)$ & $\mathrm{O}_{1}(\leq 1.3)$ & $\mathrm{O}_{1}(\leq 2.8)$ \\
\hline $\mathrm{S}_{2}(-6$ to -4$)$ & $\mathrm{O}_{2}(1.6$ to 1.7$)$ & $\mathrm{O}_{2}(1.3$ to 1.6$)$ & $\mathrm{S}_{2}(-16$ to -14$)$ & $\mathrm{O}_{2}(1.3$ to 1.4$)$ & $\mathrm{O}_{2}(2.8$ to 2.9$)$ \\
\hline $\mathrm{S}_{3}(-4$ to -2$)$ & $\mathrm{O}_{3}(1.7$ to 1.8$)$ & $\mathrm{O}_{3}(1.6$ to 1.8$)$ & $\mathrm{S}_{3}(-14$ to -12$)$ & $\mathrm{O}_{3}(1.4$ to 1.5$)$ & $\mathrm{O}_{3}(2.9$ to 3.0$)$ \\
\hline $\mathrm{S}_{4}(-2$ to 0$)$ & $\mathrm{O}_{4}(1.8$ to 2.0$)$ & $\mathrm{O}_{4}(1.8$ to 2.0$)$ & $\mathrm{S}_{4}(-12$ to -10$)$ & $\mathrm{O}_{4}(1.5$ to 1.8$)$ & $\mathrm{O}_{4}(3.0$ to 3.3$)$ \\
\hline $\mathrm{S}_{5}(0$ to 2$)$ & $\mathrm{O}_{5}(2.0$ to 2.1$)$ & $\mathrm{O}_{5}(2.0$ to 2.1$)$ & $\mathrm{S}_{5}(-10$ to -8$)$ & $\mathrm{O}_{5}(1.8$ to 2.0$)$ & $\mathrm{O}_{5}(3.3$ to 3.5$)$ \\
\hline $\mathrm{S}_{6}(2$ to 4$)$ & $\mathrm{O}_{6}(2.1$ to 2.2$)$ & $\mathrm{O}_{6}(2.1$ to 2.3$)$ & $\mathrm{S}_{6}(-8$ to -6$)$ & $\mathrm{O}_{6}(2.0$ to 2.4$)$ & $\mathrm{O}_{6}(3.5$ to 3.7$)$ \\
\hline $\mathrm{S}_{7}(4$ to 6$)$ & $\mathrm{O}_{7}(2.2$ to 2.5$)$ & $\mathrm{O}_{7}(2.3$ to 2.4$)$ & $\mathrm{S}_{7}(-6$ to -4$)$ & $\mathrm{O}_{7}(2.4$ to 2.6$)$ & $\mathrm{O}_{7}(3.7$ to 3.8$)$ \\
\hline $\mathrm{S}_{8}(6$ to 8$)$ & $\mathrm{O}_{8}(2.5$ to 2.7$)$ & $\mathrm{O}_{8}(2.4$ to 2.5$)$ & $\mathrm{S}_{8}(-4$ to -2$)$ & $\mathrm{O}_{8}(2.6$ to 2.7$)$ & $\mathrm{O}_{8}(3.8$ to 3.9$)$ \\
\hline $\mathrm{S}_{9}(8$ to 10$)$ & $\mathrm{O}_{9}(2.7$ to 2.9$)$ & $\mathrm{O}_{9}(2.5$ to 2.8$)$ & $\mathrm{S}_{9}(-2$ to 0$)$ & $\mathrm{O}_{9}(2.7$ to 2.8$)$ & $\mathrm{O}_{9}(3.9$ to 4.0$)$ \\
\hline $\mathrm{S}_{10}(>10)$ & $\mathrm{O}_{10}(>2.9)$ & $\mathrm{O}_{10}(>2.8)$ & $\mathrm{S}_{10}(>0)$ & $\mathrm{O}_{10}(>2.8)$ & $\mathrm{O}_{10}(>4.0)$ \\
\hline
\end{tabular}

Table 2. Ranges of observations and states of HMM for maximum and minimum temperatures. 


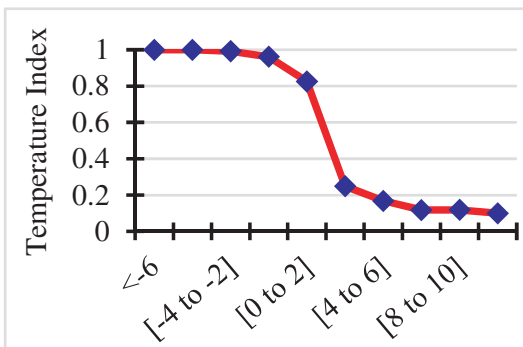

Ranges of Maximum Temperature

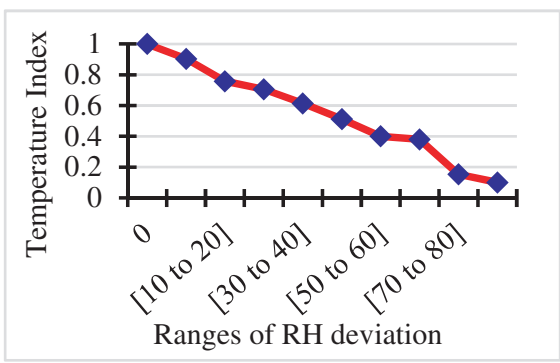

(a)

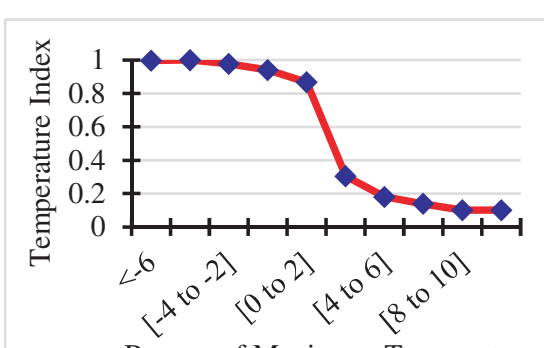

Ranges of Maximum Temperature

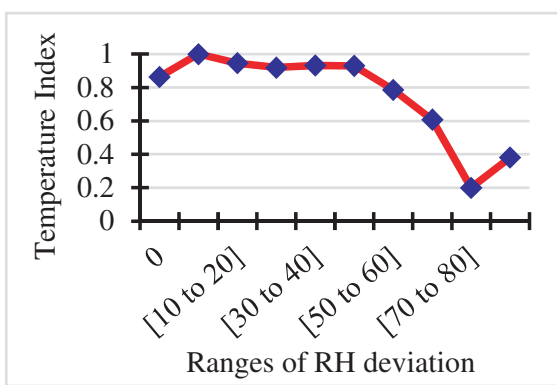

(b)
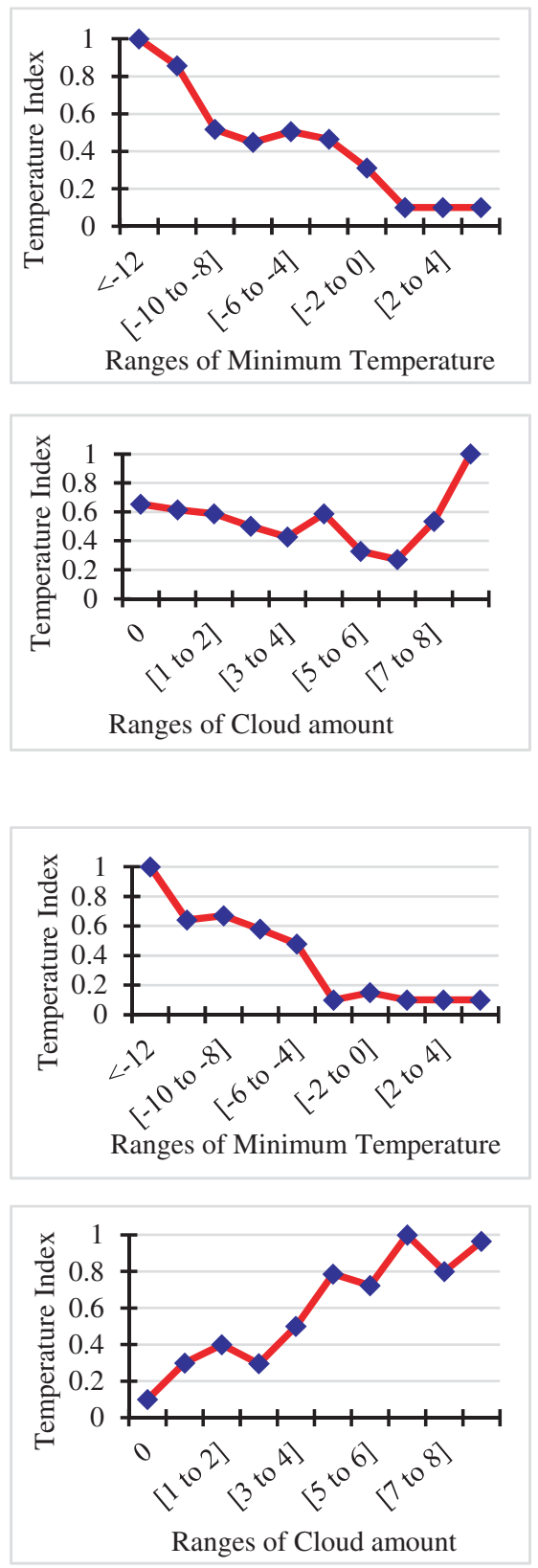
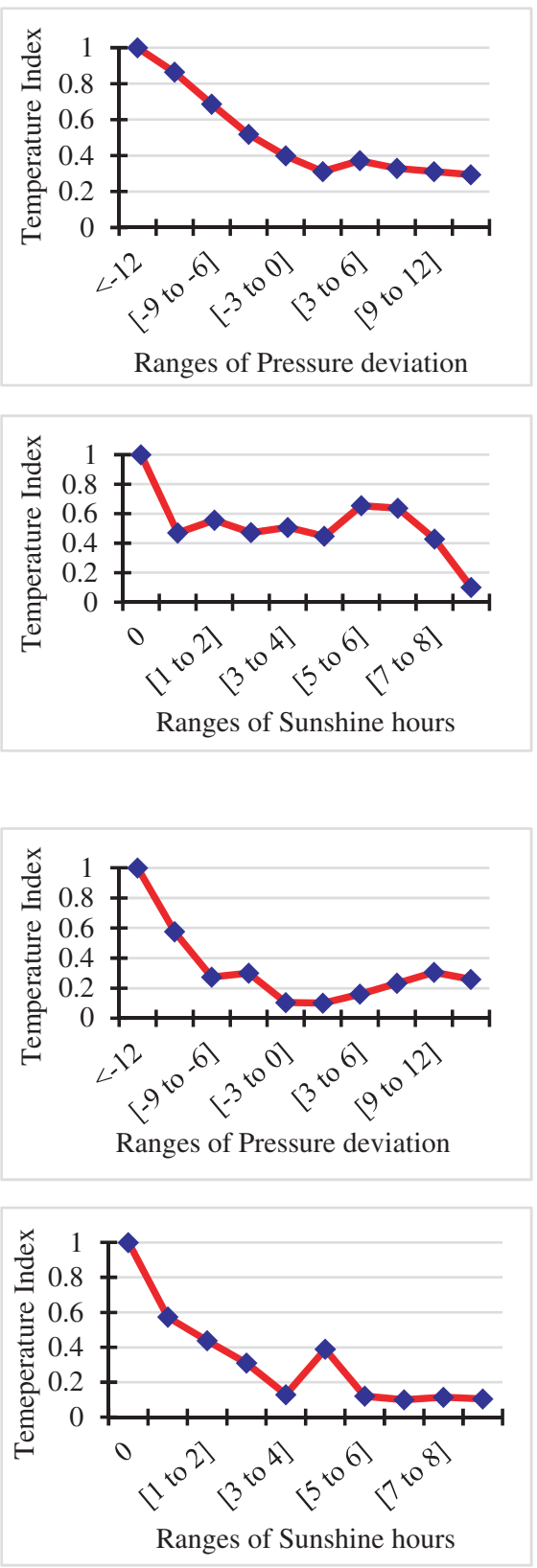

Figure 1. Plot of maximum temperature index vs. model input variables at (a) Kanzalwan and (b) Drass.

where $P$ is the probability and $t$ is the state index. This equation signifies that the probability of transition from the previous state to the current state depends on the previous state only. The HMM developed in this study is of first order (depends on the previous state only). Development of higher order HMMs involves very high computation and computational time. Most of the studies on weather forecast worldwide (Zucchini and Guttorp 1991; Hughes and Guttorp 1994; Robertson et al. 2004; etc.) are based on first order Markov models.

In the present work, the HMM is defined by 10 states and 10 observations along with three types of probabilities (state transition probabilities, observation probabilities in different states, and initial state probability). Initial state probabilities have been computed by taking the ratio of the number of counts of a state and the total number of counts. The state transition probabilities have been computed by taking the ratio of number of transitions from one state to all the other states and total number of transitions from that state to the other states. The probabilities of observations in a state have been computed by taking the ratio of number of observations and total number of observations in that state. For prediction of the most probable state sequence, most probable 


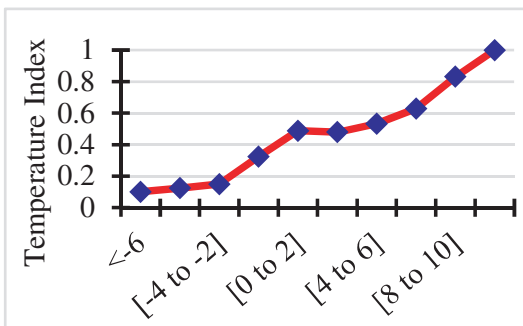

Ranges of Maximum Temperature

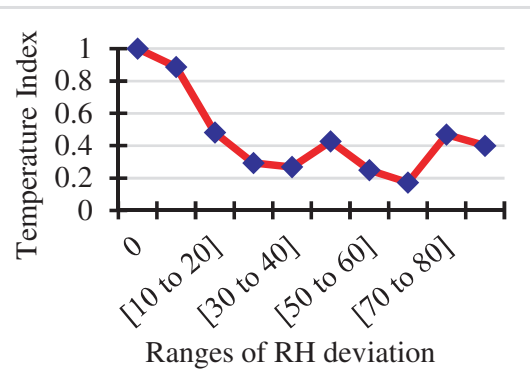

(a)
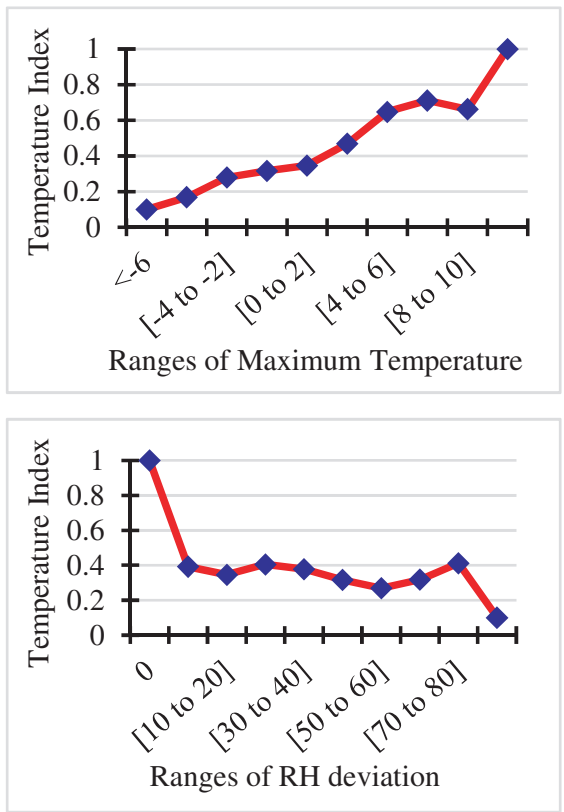
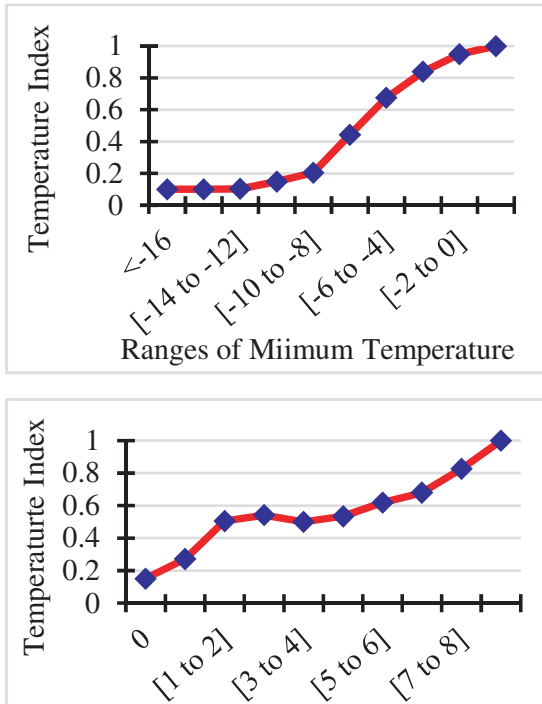

Ranges of Cloud amount
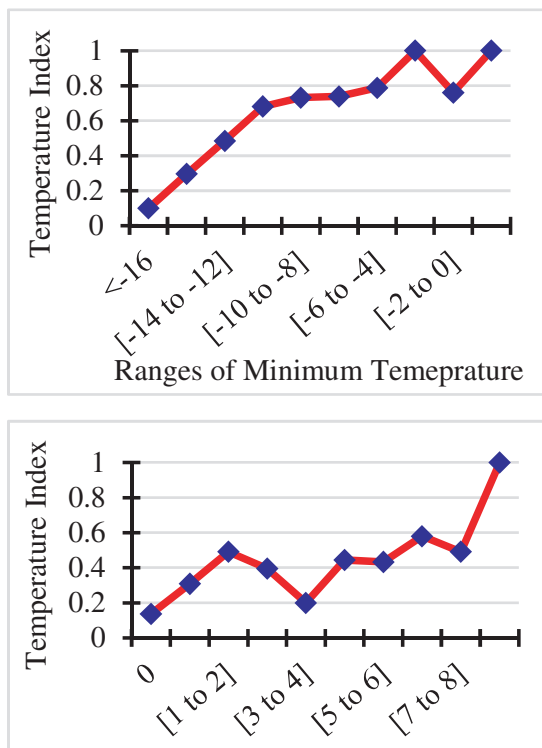

Ranges of Cloud amount
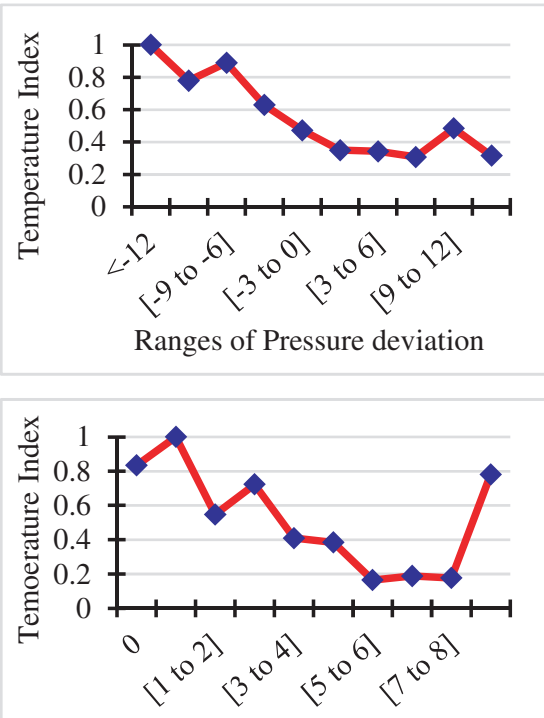

Ranges of Sunshine hours
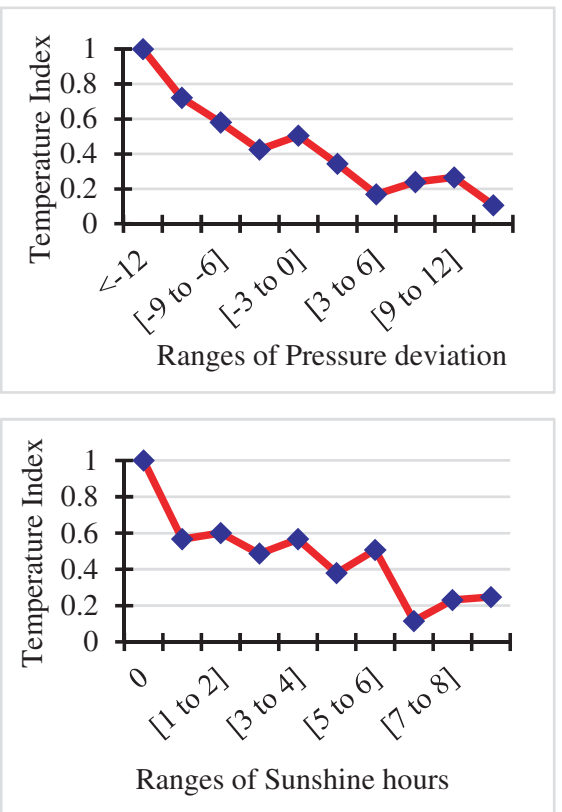

(b)

Figure 2. Plot of minimum temperature index vs. model input variables at (a) Kanzalwan and (b) Drass.

observation sequence is to be predicted. In the present model, to predict the sequence of two states, i.e., prediction for two days, a sequence of two observations has been used. The most probable observation sequence and corresponding state sequence are computed using the Forward and the Viterbi algorithms, respectively, as discussed below.

\subsection{Forward algorithm}

Forward algorithm was introduced by Rabiner (1989). It is used to compute probability of partial observation sequence, $P\left(O_{t} O_{t+1}\right)$, at state $S_{i}$ and time $t$, iteratively, as discussed in the following steps:

\section{Initialisation:}

$$
\alpha_{t}(i)=\pi_{i} * b_{i}\left(O_{t}\right), \quad 1 \leq i \leq 10
$$

Induction:

$\alpha_{t+1}(j)=\left[\sum_{i=1}^{10}\left[\alpha_{t}(i) * a i j\right]\right] * b_{j}\left(O_{t+1}\right), 1 \leq j \leq 10$

Termination:

$$
P\left(O_{t} O_{t+1}\right)=\sum_{i=1}^{10} \alpha_{t+1}(i) .
$$


Here, $\alpha_{t}(i)$ represents forward probability function at time $t$ in state $i, \pi_{i}$ represents initial state probability in state $i$ and $b_{i}\left(O_{t}\right)$ represents probability of observation $O_{t}$ in state $i$. After the most probable observation sequence has been calculated, the most probable state sequence can be calculated by using Viterbi algorithm as stated below.

\subsection{Viterbi algorithm}

Viterbi algorithm was conceived by Viterbi (1976) as a decoding algorithm for convolution codes over noisy digital communication links. It is used to compute probability of state sequence, $P\left(S_{t} S_{t+1}\right)$ corresponding to a given observation sequence, ' $O_{t} O_{t+1}$ ', iteratively, as discussed in the following steps:

Initialisation:

$$
\delta_{t}(i)=\pi_{i} * b_{i}\left(O_{t}\right), \quad 1 \leq i \leq 10
$$

\section{Induction:}

$\delta_{t+1}(j)=\operatorname{Max}\left[\delta_{t}(i) * a i j\right] * b_{j}\left(O_{t+1}\right), \quad 1 \leq j \leq 10$

Termination:

$$
P\left(S_{t} S_{t+1}\right)=\operatorname{Max}\left[\delta_{t+1}(j)\right], \quad 1 \leq j \leq 10
$$

Viterbi algorithm is similar to that of Forward algorithm, except that it uses the maximum of the function rather than summing over all the possible states. It keeps track of states in every step to predict most probable state sequence.

In HMM, it is supposed that the observed symbols and model parameters are good to explain observations, but this is not true every time. So, the model parameters are adjusted to maximise the probability of the observation sequence. To optimise model parameters, Baum-Welch algorithm, an iterative method, is used as discussed below.

\subsection{Baum-Welch algorithm}

Baum-Welch algorithm was introduced by Baum et al. (1970) in their study of statistical analysis of probabilistic functions of Markov chains. It is based on both forward and backward variables. The backward variable is the probability of the partial observation sequence from time step $t+1$ to the end. It can be calculated, iteratively, in the following steps: Initialisation:

$$
\beta_{T}(i)=1, \quad 1 \leq i \leq 10
$$

Induction:

$$
\begin{aligned}
& \beta_{t}(i)=\sum a_{i j} * b_{j}\left(O_{t+1}\right) * \beta_{t+1}(j), \\
& 1 \leq t \leq 9,1 \leq j \leq 10 \text {. }
\end{aligned}
$$

From the forward and backward variables, two new variables can be calculated. The first one is $\xi_{t}(i, j)$, the probability of being in state $S_{i}$ at time $t$, and state $S_{j}$ at time $t+1$, given the model and the observation sequence, i.e,

$$
\begin{aligned}
\xi_{t}(i, j)= & \alpha_{t}(i) \times a_{i j} \times b_{j}\left(O_{t+1}\right) \\
& \times \beta_{t+1}(j) / P(O / \lambda) .
\end{aligned}
$$

The second one is $\gamma_{t}(i)$, the probability of being in state $S_{i}$ at time $t$, given the observation sequence $O$, and the model, i.e.,

$$
\gamma_{t}(i)=\alpha_{t}(i) \times \beta_{t}(i) / P(O / \lambda) .
$$

The variables $\xi$ and $\gamma$ satisfy the relationship

$$
\gamma_{t}(i)=\sum \xi_{t}(i, j), \quad 1 \leq j \leq 10 .
$$

Now, if $\gamma_{t}(i)$ is summed over all instants (excluding instant $T$ ), the expected number of times the state $S_{i}$ has been visited is obtained. On the other hand, if $\xi_{t}(i, j)$ is summed over all instants (excluding instant $T$ ), the expected number of transitions that have been made from state $i$ to $j$ is obtained. From this behaviour of $\gamma_{t}(i)$ and $\xi_{t}(i, j)$, the following re-estimations of the model parameters could be deduced:

$$
\begin{gathered}
\pi^{\prime}=\gamma_{1}(i), \\
a_{i j}^{\prime}=\sum_{t=1}^{T-1} \xi_{t}(i, j) / \sum_{t=1}^{T-1} \gamma_{t}(i), \\
b_{i j}^{\prime}(k)=\sum_{\substack{t=1 \\
O t=w k}}^{T} \gamma_{t}(i) / \sum_{t=1}^{T} \gamma_{t}(i) .
\end{gathered}
$$

After re-estimations of the model parameters, a new model $\lambda^{\prime}$ is obtained, which is more likely than model $\lambda$, producing observation sequence $O$. This process of re-estimation is continued till no improvement in the probability of observation sequence reached.

\section{Results and discussion}

HMMs have been developed for prediction of maximum and minimum temperatures in two different mountain ranges of the Himalaya. The models use nine meteorological variables as input for prediction of the maximum and minimum temperatures, with a lead time of two days. The plots of input variables of the models with their TIs for maximum and minimum temperatures are shown in figures 1 and 2, respectively. The RMSE of the models for maximum and minimum temperatures 
with and without optimisation has been compared with the RMSE of persistence temperature forecasts as well as the standard deviation of maximum and minimum temperatures for both the stations as shown in figures 3 and 4 . The long-term mean and standard deviation (1992-2012) of maximum and minimum temperature for Kanzalwan and Drass are shown in table 1.

The plots of the input variables and their TIs for maximum temperature (figure 2) reveal that, both in lower and middle climatic zones of the Himalaya, the temperature index is higher for initial categories of the variables - maximum temperature, minimum temperature, pressure deviation, relative humidity deviation and sunshine hours. As the value of the variable increases in the category, the TI decreases. In the case of cloud amount, TI increases with the increase of the value of the variable. When the value of the variables - maximum temperature, minimum temperature, pressure deviation, relative humidity deviation and sunshine hours increases, the TI, i.e., the number of days having a temperature lower than mean maximum temperature decreases. When the amount of clouds increase, the number of days having maximum temperature lower than mean maximum temperature increases and hence the TI.

The plots of the input variables and their TIs for minimum temperature (figure 1) signifies that in both, the Pir-Panjal and Great Himalayan mountain ranges of Indian Himalaya, the TI is initially smaller for maximum temperature, minimum temperature and cloud amount and increases with the increase of these variables. The variables pressure deviation, relative humidity deviation and sunshine hours show a higher initial value of temperature index, which decreases as the value of the variable increases. In the case of maximum temperature, minimum temperature and cloud amount, the number of days having the minimum temperature higher than mean minimum temperature increases thus increasing temperature index. For the variables - pressure deviation, relative humidity deviation and sunshine hours, the number of days having the minimum temperature higher than mean minimum temperature decreases thus decreasing temperature index.

The RMSE analysis of the models with optimisation reveals that the model for maximum temperature has shown $4 \%$ and $12 \%$ improvement
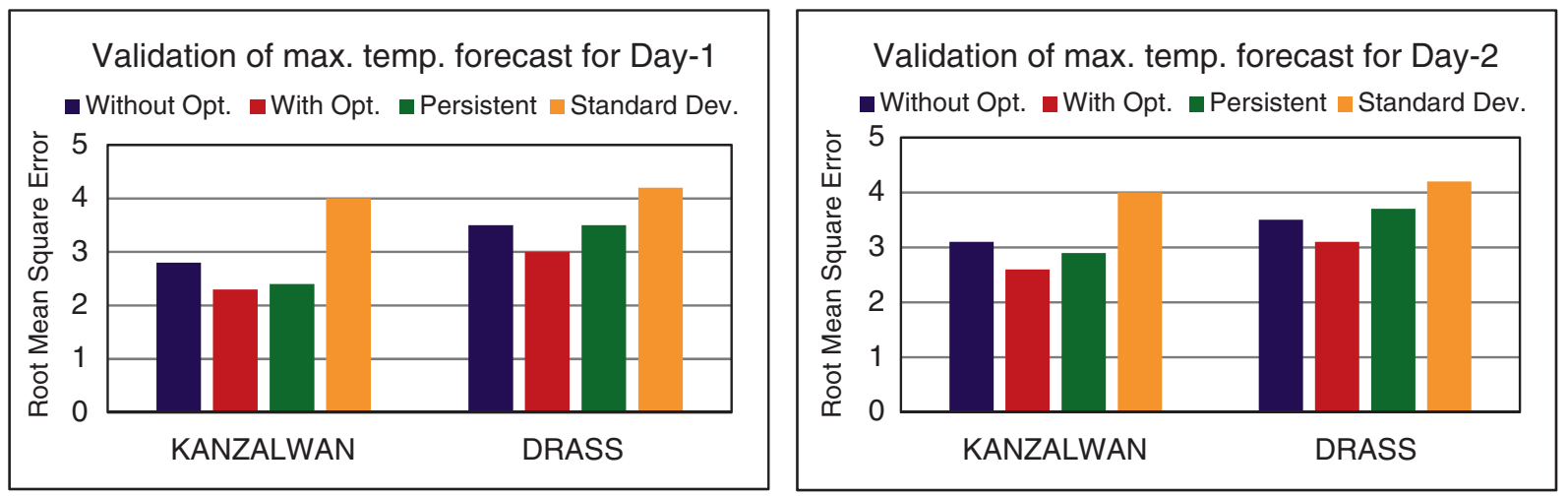

Figure 3. Validation of forecast for maximum temperature.
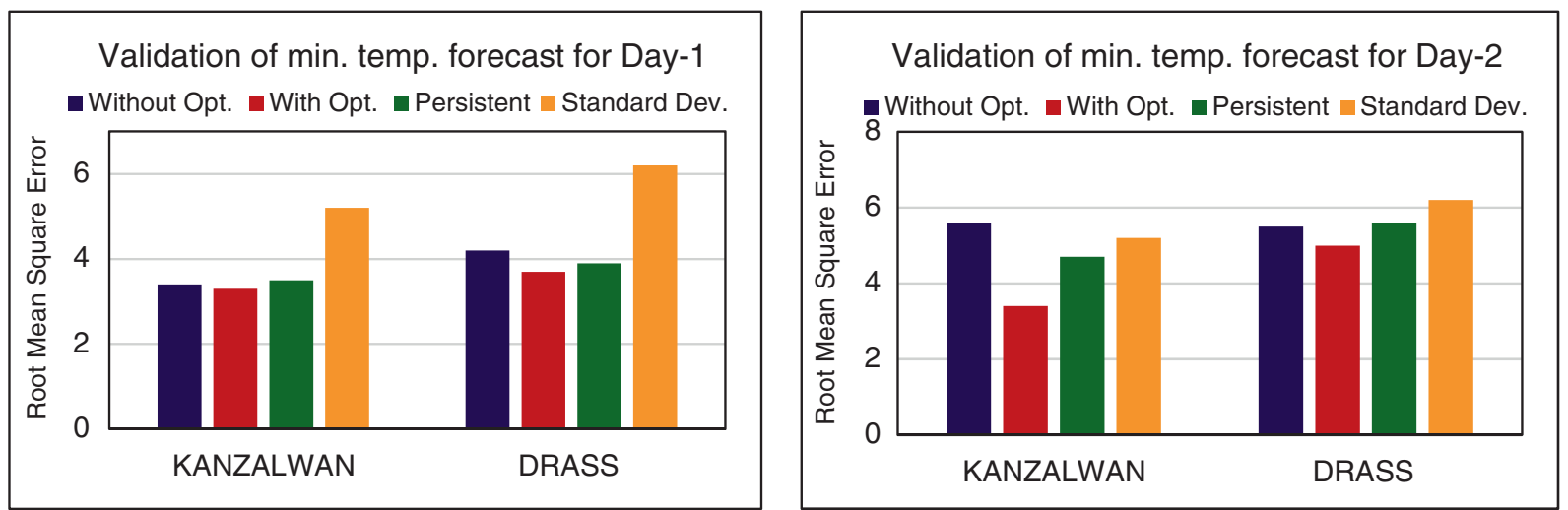

Figure 4. Validation of forecast for minimum temperature. 
in the forecast over persistence forecast for day-1 and $17 \%$ and $19 \%$ for day-2 for Kanzalwan and Drass, respectively. For minimum temperature, it has shown a $6 \%$ and $38 \%$ improvement for day-1 and $5 \%$ and $12 \%$ for day-2. In the study by Dimri et al. (2002), the RMSE of maximum and minimum temperature predictions for Stage-II is within $\pm 3^{\circ} \mathrm{C}$, which is less than or equal to the standard deviation. In the present study, the RMSE of maximum temperature for Kanzalwan is less than $3^{\circ} \mathrm{C}$ and for Drass, it is less than $3.2^{\circ} \mathrm{C}$ for both the days, which is less than the standard deviation (figures 3 and 4). The RMSE of minimum temperature for Kanzalwan is $<3.5^{\circ} \mathrm{C}$, and for Drass, it is less than or equal to $5^{\circ} \mathrm{C}$ for both the days which is less than the standard deviation. As far as only one station in Pir-Panjal range of the Himalaya is concerned, the HMM performance is better. Moreover, the HMM is able to forecast with an increased lead time of two days. The RMSE reduction with the optimised HMM is less than half a degree than the persistence forecast. However, the persistence forecast is not reliable due to its failure at the transition of weather states. Therefore, an incremental improvement in the forecast of maximum and minimum temperatures over persistence forecast is useful and can be applied reliably as an input in other important studies, such as avalanche forecasting.

\section{Conclusion}

In this study, Hidden Markov Models have been developed for forecasting of maximum and minimum temperatures for Kanzalwan in Pir-Panjal and Drass in Great Himalayan mountain range of Indian Himalaya. The models provide forecast with a lead time of two days. The RMSE of the models has been compared with that of persistence forecast. Comparison of RMSE of the model and persistence forecast reveal that the RMSE of the optimised model has been found smaller than the persistence forecast for both the stations in Indian Himalaya. Though the reduction in RMSE by optimisation is small, it is reliable than persistence forecast and can be used in other important studies such as avalanche forecasting. This study can further be extended to other parts of the Himalaya for its use in avalanche forecasting, defence operations and civil population for the planning of outdoor activities and to assess the severity of the cold conditions during winter. The models developed in this study are purely based on a historical database of surface observations and can further be improved by incorporating large scale synoptic observations to reduce forecasting errors associated with the changing synoptic weather conditions.

\section{Acknowledgements}

The authors acknowledge all the reviewers of this paper for the valuable suggestions and positive comments. Director, SASE is duly acknowledged for providing support and encouragement. Field data collection teams of Snow and Avalanche Study Establishment are also acknowledged for collecting data in adverse climatic conditions of the Himalaya during winter.

\section{References}

Attri S D, Pandya A B and Dubey D P 1995 Forecasting of minimum temperature over Gangtok; Mausam 46 63-68.

Baum L E, Petrie T, Soules G and Weiss N 1970 A maximum technique occurring in the statistical analysis of probabilistic functions of Markov chains; Ann. Math. Stat. 41(1) 164-171.

Bicego M, Castellani U and Murino V 2003. Using Hidden Markov Models and wavelets for face recognition; Proc. 12th Int. Conf. Imag. Analysis Process.

Bhusari V and Patil S 2011 Application of Hidden Markov Model in credit card fraud detection; Int. J. Distrib. Parall. Syst. 2(6) 203-211.

Bolch T, Kulkarni A, Kääb A, Huggel C, Paul F, Cogley J G, Frey H, Kargel J S, Fujita K, Scheel M, Bajracharya S and Stoffel M 2012 The state and fate of Himalayan glaciers; Science 336 310-314.

Chrantorois T and Liakatas A 1990 Study of minimum temperatures employing Markov chains; Mausam 41 69-74.

Dimri A P, Mohanty U C and Ravi N 2002 Statistical model based forecast of minimum and maximum temperature at Manali; Curr. Sci. 82 997-1003.

Dimri A P, Mohanty U C and Rathore LS 2005 Minimum temperature forecast at Manali, India; Curr. Sci. 88(4) 927-934.

Gales M and Young S 2007 Application of Hidden Markov Model in speech recognition; Found. Trends Sign. Process. 1(3) 195-304.

Hughes J P and Guttorp P 1994 Incorporating spatial dependence and atmospheric data in a model of precipitation; J. Appl. Meteorol. 33 1503-1515.

Joshi J C and Srivastava S 2014 A Hidden Markov Model for avalanche forecasting on Chowkibal-Tangdhar road axis in Indian Himalayas; J. Earth Syst. Sci. 123(8) 17711781.

Maini P, Kumar A, Singh S V and Rathore L S 2002 Statistical interpretation of NWP products in India; Meteorol. Appl. 9(1) 21-31.

Mohan V, Jargle N K and Kulkarni P D 1989 Numerical prediction of daily maximum temperature over Ozar; Mausam 40 227-228.

Mohanty U C, Ravi N, Madan O P and Paliwal R K 1997 Forecasting minimum temperature during winter and maximum temperature during summer at Delhi; Meteorol. Appl. 4(1) 37-48.

Kumar A and Maini P 1996 Statistical interpretation of general circulation model: A prospect for automation of medium range local weather forecast in India; Mausam 47(3) 229-236.

Rabiner L R 1989 A tutorial on Hidden Markov Model and selected applications in speech recognition; Proceedings of IEEE 73(2).

Raj Y E A 1989 Prediction of winter minimum temperature at Pune by analogue and regression method; Mausam 40 $175-180$. 
Raj Y E A 1998 On forecasting daily minimum temperature at Madras; Mausam 49 95-102.

Robertson A W, Kirshner S and Smyth P 2004 Downscaling of daily rainfall occurrence over Northeast Brazil using a hidden Markov model; J. Climate 17(22) 4407-4424.

Sanjeeva Rao P, Saseendran S A, Rathore L S and Bahadur 1996 Medium range weather forecasts in India during monsoon-1994; J. Meteorol. Appl. 3 317-324.

Singh D, Ganju A and Singh A 2005 Weather prediction using nearest-neighbor model; Curr. Sci. 88(8) 1283-1289.

Tanguay D O 1995 Hidden Markov Model for gesture recognition; ME Thesis, Massachusetts Institute of Technology.

Vashisht R C and Pareek R S 1991 Forecasting of minimum temperature at Jaipur and south Rajasthan; Mausam 42 113-116.
Viterbi A J 1976 Error bounds for convolutional codes and an asymptotically optimum decoding algorithm; IEEE Trans. Info. Theory 13(2) 260-269.

Woodcock F 1984 Australian experimental model output statistics forecasts of daily maximum and minimum temperature; Mon. Wea. Rev. 112 2112-2121.

Xuan T 2004. Autoregressive Hidden Markov Model with application in an El-Nino study; MS Thesis, University of Saskatchewan Saskatoon.

Zhang Y 2004. Prediction of financial time series with Hidden Markov Models; MS Thesis, Simon Fraser University.

Zucchini W and Guttorp P 1991 A Hidden Markov Model for space-time precipitation; Water Resour. Res. 27 19171923.

MS received 29 December 2015; revised 19 September 2016; accepted 13 October 2016

Corresponding editor: A K SAHAI 